\title{
Properties and Exactions of Essential Oil from the Fruit of Clausena Lansium(Lour.) Skeels in Zhaoqing Area
}

\author{
Qiaohui Liang ${ }^{1}$, Peijun Zheng ${ }^{1}$, Rongrong $\mathrm{Xu}^{1}$, Deqiu Xie ${ }^{2}$, Mingyin $\mathrm{Xu}^{2}$, Chongbi Li ${ }^{2 *}$ \\ ${ }^{1}$ Zhaoqing University Undergraduates, Biotechnology Professional Students \\ ${ }^{2}$ Zhaoqing Medicine College. Zhaoqing City \\ *chongbili@163.com
}

\begin{abstract}
The water vapor distillation method was used for extracting the volatile oil in Wampee fruits, the volatile oil was identified by thin layer chromatography $(T L C)$, and the component extraction for volatile oil was determined by GCMS computer combined with identification instrument the composition and the peak area normalization method to get the relative content of each component. Results showed that the 25 peaks isolated were identified 23 components which are phellandrene (36.97\%), terpinen-4-ol (17.58\%), sabinene hydrate (13.76\%). These three substances are relatively high. In the study of the antioxidant activity of the volatile oil, the effect of the scavenging rate of hydroxyl radical was studied. It was found that the concentration of volatile oil in a certain concentration range was positively correlated with the clearance rate of hydroxyl radical. The filter paper method for study the antimicrobial activity of the essential oil presented that it all had a certain antibacterial effect to Staphylococcus aureus, Bacillus subtilis and Escherichia coli. And inhibition of different concentrations of essential oils was different for different types of bacteria. This bacteriostasis becomes smaller as with the decrease of concentration. And the effect of essential oil on mouse pancreatic cancer cells was also studied. The experiments showed that the essential oil had an effect of tumor inhibition.
\end{abstract}

Keyword: Fruit of clausena lansium(Lour.) skeels, essential oil;chemical components, properties

\section{INTRODUCTION}

Wampee, one of the popular tropical fruit is Rutaceae evergreen small trees or large shrubs and has a high nutritional value. This fruit has a high medicinal value including the leaf, fruit and seeds as medicine, It could help stomach health and digestion, relieve thirst, gas, cough, pain and other effects(LI Ruizhen et al, 2007 ), Wampee fruit is rich in amino acids, sugars, organic acids, pectin, vitamin C, essential oils, flavonoids and other effective components(Tang Wenning et al.,2002 ).Zhaoqing also has many of the specific Wampee in China, and Wampee seeds are rich of oil belonging to the traditional chinese medicine volatile oil with a variety of biological activities as antibacterial, anti-inflammatory, anti-cancer, analgesic, diuretic and so on (Jiang Zitao et al.,2012). Therefore, the research on the volatile components and the development of its medicinal value would have an important significance. Volatile oil from Clausena lansium used making perfume process has not been reported up to now. So fitting of essential oil being made of perfume from wampee fruit is necessary for the antioxidant and inhibitory effects cancer effect analysis, It would provide a theoretical and experimental basis for the study of yellow fruit essential oil and also have a profound significance on the development prospect and application development.

\section{Material AND MethodS}

\subsection{Extraction of Volatile Oil}

The volatile oil extractor was used in yellow volatile oil extraction. As follows, $100 \mathrm{~g}$ fresh fruit of chinese wampee were added distilled water for homogenation, and ground with a micro-plant grinding machine (FZ102; Tianjin Taisite Instruments, Tianjin, China) and then were extracted for $6 \mathrm{~h}$ in volatile oil extractor. The fluid extracted including oil-water was divided by using high speed refrigerated centrifuge (Eppendorf). The fluids adding a little of anhydrous sodium sulfate were filtered by using a $0.45 \mathrm{um}$ membrane filter. At last a transparent, colorless volatile oil was got. 


\subsection{Chromatographic Analysis}

The yellow volatile oil identification would be used by TLC method (Zhang Zhi et al, 2009). The volatile oil comparison was analyzed in mass spectrometry (Shimadzu QP2010GCMS). The parameters as follows, chromatographic column for RTX-5MS, quartz capillary column $(30 \mathrm{~m} * 0.25 \mathrm{~mm} * 0.25 \mathrm{um})$, Ion source for EI source, at a temperature of 230 degrees, the electronic energy $70 \mathrm{eV}$, the interface temperature of 230 degrees, solvent delaying for $4 \mathrm{~min}$, scanning range 30 600amu. After heating program $60 \square$, carrier gas for helium, column flowing speeds for $1.5 \mathrm{ml} / \mathrm{min}$, sample quantity for $1 \mathrm{ul}$, split-flow ratio of 10:1, staying for $5 \mathrm{~min}$ and then to 4 degree $/$ min increasing up to $250 \quad \mathrm{C}$, retention for $7 \mathrm{~min}$.

\subsection{Preparation of the Perfume}

$8 \%$ of the essential oils which included $3.6 \%$ of volatile oil from Clausena, $2.4 \%$ of essential oil of lavender (bought, made in China) and 2.0\% sandalwood essential oil (bought, made in China) and ethanol of $92 \%$ would be mixed according to a certain proportion, After 2 hours of ultrasonic emulsified, the insoluble impurities were removed by $6000 \mathrm{~g}$ centrifugation at $4 \mathrm{C}$ for 10 minutes to get the clarified liquid perfume.

\subsection{Scavenging Hydroxyl Radical}

The Yellow volatile oil properties were determined (Jiang Zitao et al,2012,Chen Sijia et al,2015,Zhang Fuping et al, 2013), Preparation of volatile oil solution were diluted with $60 \%$ as solvent into $1,2,4,8,16 \mathrm{mg} / \mathrm{ml}$ of volatile oil solution and $2.502 \mathrm{~g}$ of ferrous sulfate (Sigma) $7 \mathrm{H} 2 \mathrm{O}$ were dissolved in $1000 \mathrm{ml}$ of deionized water and prepared for $9 \mathrm{mmol} / \mathrm{L}$ of ferrous sulfate solution and placed in the dark. Additionally, 1.243g salicylic acid crystal (Sigma) will be dissolved into $1000 \mathrm{ml}$ with ethanol solution, and $9 \mathrm{mmol} / \mathrm{L}$ salicylic acid- ethanol solution was prepared. Then the solution would be again dissolved in peroxide hydrogen of $30 \%$ about $889 \mathrm{ml}$ into $1000 \mathrm{ml}, 8.8 \mathrm{mmol} / \mathrm{L}$ of hydrogen peroxide solution for use.

\subsection{Determination of Hydroxyl Free Radical Scavenging Rate of Volatile Oil}

$1 \mathrm{ml}$ of $9 \mathrm{mmol} / \mathrm{L}$ ferrous sulfate solution, $1 \mathrm{ml}$ of $9 \mathrm{mmol} / \mathrm{L}$ salicylic acid ethanol solution, $1 \mathrm{ml}$ of different concentrations of samples were mixed, then the $1 \mathrm{ml}$ of $8.8 \mathrm{mmol} / \mathrm{L}$ hydrogen peroxide was added finally for reaction30min at $37{ }^{\circ} \mathrm{C}$ as a control of distilled water. The OD value of each concentration was measured at a 510nm of wavelength in spectrophotometer (type 722, China). The measurements were repeated three times, and the average values were got as the measured results. The formula on calculation $\mathrm{OH}$ clearance rate as follows.

A0 - absorbance of blank control solution.

Ax - absorbance after adding sample solution.

Ax0 - no color reagent $\mathrm{H} 2 \mathrm{O} 2$ only the optical absorption of sample solution.

volatile oil solution preparation (dichotomy)

Oil wampee pure oil obtained after filtering the water (anhydrous sodium sulfate) was diluted with acetone into7 different concentrations as $10 \%, 5 \%$ and $2.5 \%, 1.25 \%, 0.625 \%, 0.3125 \%$ and $0.156 \%$ respectively.

\subsection{Antibacterial Activity of the Essential Oil}

The antimicrobial activities of the essential oil were tested against three different bacteria. Two Gram-positive strains were Staphylococcus aureus and Bacillus subtilis, one Gram-negative strain was Escherichia coli .Staphylococcus aureus, Bacillus subtilis and Escherichia coli are three of common bacteria. These three kinds of bacteria are common and easy to cultivate, so we can use these three kinds of bacteria to explore the antibacterial activity of the perfume. The strains were provided by the Centre of Biopharmaceutical Engineering in Zhaoqing University. The bacteria incubation was placed for growth in the shelter to save the light. In order to make the experimental result standard and accurate, the method of the turbidity of wheat to measure the concentration of the diluted liquid were used. The bacteria solution was $1 \mathrm{ml}$, and the bacterial concentration was about $1.5 \times 10^{\wedge} 8 \mathrm{cfu} / \mathrm{ml}$, which was diluted with normal saline to the turbidity and 0.5 wheat's standard tubes. And the essential oil from Zhaoqing wampee needs to be filtered. Then the antibacterial activity was investigated by agar disc diffusion method which a hole was punched into a $6 \mathrm{~mm}$ filter paper in diameter. The micro broth 
dilution was as follows $2 \times 106 \mathrm{CFU} / \mathrm{mL}$. A control was also performed in containing inoculated broth supplemented with only DMSO. The maximum final concentration of DMSO was $2 \%$ in each medium which the test strains were incubated at $37^{\circ} \mathrm{C}$ for $24 \mathrm{~h}$. Then the size of inhibition ring was examined for evidence of antimicrobial (Zhang Chunle et al, 2006, Su Xiufang et al, 2011.).

\subsection{Detection of the Cell Proliferation Inhibition the Perfume by MTT Method}

The human colon carcinoma LoVo cells in DMEM culture medium containing $10 \%$ fetal bovine serum, $100 \mathrm{u} / \mathrm{ml}$ penicillin and $100 \mathrm{u} / \mathrm{ml}$ streptomycin) culture, were placed in $37{ }^{\circ} \mathrm{C}, 5 \% \mathrm{CO} 2$ and $80 \%-90 \%$ of humidity in the incubator, the medium was replaced once every 2-3 days for daily observation of cell grow, and then they were adjusted the cell for $5 \times 106 / \mathrm{ml} \sim 1 * 107$ in density. The cells in Logarithmic growth phase were discarded the old medium, and washed with 2-3ml PBS washing twice, then was degraded with $0.25 \%$ trypsin for $2 \mathrm{~min}$, adding complete medium termination of digestion and spheroid cells into single cells (under inverted microscope observation), counting, cell concentration was adjusted to 40000-60000 / $\mathrm{ml}$. The suspended cells were seeded in three of 96 well plates, the cells of each plate were divided into 8 lines for testing. Each line for five wells, including control line (line 1), the experimental wellswere5lines, 10 wells each line, and the control line for five wells zero adjustments, $100 \mathrm{ul}$ of each well, only adding a complete incubating medium. All the plates were incubated at $37 \mathrm{C}, 5 \% \mathrm{CO} 2$ and under the condition of $80 \%-90 \%$ humidity in $\mathrm{CO} 2$ incubator. The morphology and growth of cells were observed every day. After 24 hours until the cells adhered to the wall, the experimental wells were added 100ul of perfume (ling 2, line 3, line 4 and line 5. and the control wells were added 100ul of 95\% ethanol including the holes in line 6, line 7 and line 8 of the plate. The supernatant was sucked out in the super clean working table, and 20ul of MTT solutions of $0.5 \mathrm{mg} / \mathrm{ml}$ were added to each well of the plate, placed in 37 degrees Celsius, $5 \% \mathrm{CO} 2$ and humidity of $80 \%-90 \%$ to continue cultivating. The growth and morphology of the cancer cells experimented were observed and counted at $0,1,4,18,24 \mathrm{~h}$ of different time under the inverted microscope. The supernatants in the plates were sucked out gently and added into 150uLDMSO solutions in each hole for shaking 5-10 minutes gently, after standing for 5 minutes, absorbance values were examined at $490 \mathrm{~nm}-630 \mathrm{~nm}$ of wavelength on the enzyme mark analyzer and repeated the examination twice. (Xing Yan Bian, 1998).

The inhibition rates were calculated according to the following formula.

The inhibition rate $=(\mathrm{OD}$ value of the cell control hole $-\mathrm{OD}$ value of the experimental hole $/ \mathrm{OD}$ value of the control hole $\mathrm{x} 100 \%$

\subsection{Statistical Methods}

Measurement data were conducted by using the methods F deviation and LSD (Xiao-Hua Zhou, et al,2011 )

\section{RESUltS}

\subsection{Preparation of Volatile Oil}

The extracting rates on zhaoqing yellow fruit are $0.4 \%$ by using steam distillation. The volatile oil was identified by thin plate chromatography method (Fig 1).

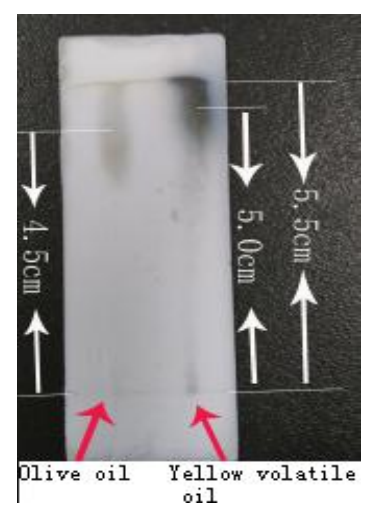

Fig1. Thin plate chromatography identification of essential oils 
Qiaohui Liang et al.

\subsection{Components Analysis}

The various components were identified according to the parameters designed and the peaks spectrum obtained by scanning the mass. The relative percentage contents of each component were got by NIST27 and NIST147 mass spectrometry spectra library and the artificial analysis with control, It was got that 25 components from the characteristics of Zhaoqing yellow fruits were separated and 23 components including terpenes and alcohols were identified. And the content of the highest is beta phellandrene, accounting for $36.97 \%$, secondly another both of terpinen-4-ol and sabinene hydrate, accounting for the $17.58 \%$ and $13.76 \%$ respectively (Table 1 ).

Table1. Composition analysis of yellow peel naphtha

\begin{tabular}{|c|c|c|c|c|c|}
\hline Number & $\begin{array}{l}\text { retention time } \\
(\mathrm{min})\end{array}$ & chemical name & $\begin{array}{l}\text { chemical } \\
\text { formula }\end{array}$ & $\begin{array}{l}\text { molecular } \\
\text { weight }\end{array}$ & $\begin{array}{l}\text { relative percentage } \\
\text { content } / \%\end{array}$ \\
\hline 1 & 6.109 & $\alpha$ - cedar ene & $\mathrm{C} 10 \mathrm{H} 16$ & 136 & 0.27 \\
\hline 2 & 6.354 & $\alpha$-pinene & $\mathrm{C} 10 \mathrm{H} 16$ & 136 & 4.76 \\
\hline 3 & 7.811 & Juniper ene & $\mathrm{C} 10 \mathrm{H} 16$ & 136 & 13.76 \\
\hline 4 & 7.907 & $\beta$-Beta pinene & C10H16 & 136 & 0.46 \\
\hline 5 & 8.451 & $\beta$-Beta myrcene & C10H16 & 136 & 2.48 \\
\hline 6 & 8.948 & $\begin{array}{l}\alpha \text {-Alpha water } \\
\text { dropwort ene }\end{array}$ & C10H16 & 136 & 4.26 \\
\hline 7 & 9.423 & (+)-4- carene (+) - & $\mathrm{C} 10 \mathrm{H} 16$ & 136 & 3.01 \\
\hline 8 & 9.758 & cymene & $\mathrm{C} 10 \mathrm{H} 14$ & 134 & 2.56 \\
\hline 9 & 9.988 & $\begin{array}{l}\beta-- \text { water dropwort } \\
\text { ene }\end{array}$ & C10H16 & 136 & 36.97 \\
\hline 10 & 11.090 & terpinene & C10H16 & 136 & 5.19 \\
\hline 11 & 12.224 & 2-- carene & $\mathrm{C} 10 \mathrm{H} 16$ & 136 & 1.22 \\
\hline 12 & 12.726 & linalool & $\mathrm{C} 10 \mathrm{H} 18 \mathrm{O}$ & 154 & 0.14 \\
\hline 13 & 13.545 & $\begin{array}{l}1 \text { - methyl - } 4 \text { - (1 - } \\
\text { methyl ethyl) - anti - } \\
2 \text { - cyclohexene - } 1 \text { - } \\
\text { alcohol }\end{array}$ & $\mathrm{C} 10 \mathrm{H} 18 \mathrm{O}$ & 154 & 0.75 \\
\hline 14 & 14.253 & $\begin{array}{l}1 \text { - methyl - } 4 \text { - (1 - } \\
\text { methyl ethyl) - shun - } \\
2 \text { - cyclohexene - } 1 \text { - } \\
\text { alcohol }\end{array}$ & $\mathrm{C} 10 \mathrm{H} 18 \mathrm{O}$ & 154 & 0.47 \\
\hline 15 & 15.732 & 4- terpene alcohols & $\mathrm{C} 10 \mathrm{H} 18 \mathrm{O}$ & 154 & 17.58 \\
\hline 16 & 16.096 & Implicit ketone & $\mathrm{C} 9 \mathrm{H} 14 \mathrm{O}$ & 138 & 0.48 \\
\hline 17 & 16.197 & $\alpha$ - terpineol & $\mathrm{C} 10 \mathrm{H} 18 \mathrm{O}$ & 154 & 0.76 \\
\hline 18 & 16.826 & Cis menthol & $\mathrm{C} 10 \mathrm{H} 18 \mathrm{O}$ & 154 & 0.19 \\
\hline 19 & 17.847 & unidentification & & & 0.14 \\
\hline 20 & 20.890 & unidentification & & & 0.27 \\
\hline 21 & 24.031 & $\beta$ - caryophyllene & $\mathrm{C} 15 \mathrm{H} 24$ & 204 & 1.22 \\
\hline 22 & 26.799 & $\beta$ - red myrrh ene & $\mathrm{C} 15 \mathrm{H} 24$ & 204 & 1.50 \\
\hline 23 & 27.264 & $\beta$-half water dropwort & $\mathrm{C} 15 \mathrm{H} 24$ & 204 & 0.15 \\
\hline 24 & 27.508 & $\alpha$-sweet limonene & $\mathrm{C} 15 \mathrm{H} 24$ & 204 & 1.21 \\
\hline 25 & 31.819 & santalol & $\mathrm{C} 15 \mathrm{H} 24 \mathrm{O}$ & 220 & 0.22 \\
\hline
\end{tabular}

\subsection{Influence of Hydroxyl Free Radical Clearance on the Oil}

The perfume from chinese wampee fruit and commercially available perfume were diluted into different concentrations gradient to determine the ability of hydroxyl radical scavenging for the two kinds of perfume. It was found that the hydroxyl removal rate of yellow pear perfume was better than the selection of commercially available perfume, The determination of volatile oil of hydroxyl free radical clearance rate showed that hydroxyl free radical clearance rate curve was increasing in the range of 1 to $16 \mathrm{mg} / \mathrm{ml}$ with the increase in the concentration, and the maximum clearance rate was $90.8 \%$ in concentration of $16 \mathrm{mg} / \mathrm{ml}$ (see table 2 and figure 1 ). 
Properties and Exactions of Essential Oil from the Fruit of Clausena Lansium(Lour.) Skeels in Zhaoqing Area

Table2. Free radical clearance measurement of volatile oil of different concentrations

\begin{tabular}{|l|l|l|l|l|l|}
\hline sample con $(\mathrm{mg} / \mathrm{ml})$ & 1 & 2 & 4 & 8 & 16 \\
\hline clearence \% & 13.9 & 28.1 & 51.5 & 69.8 & 90.6 \\
\hline
\end{tabular}

Hydroxyl free radical clearance

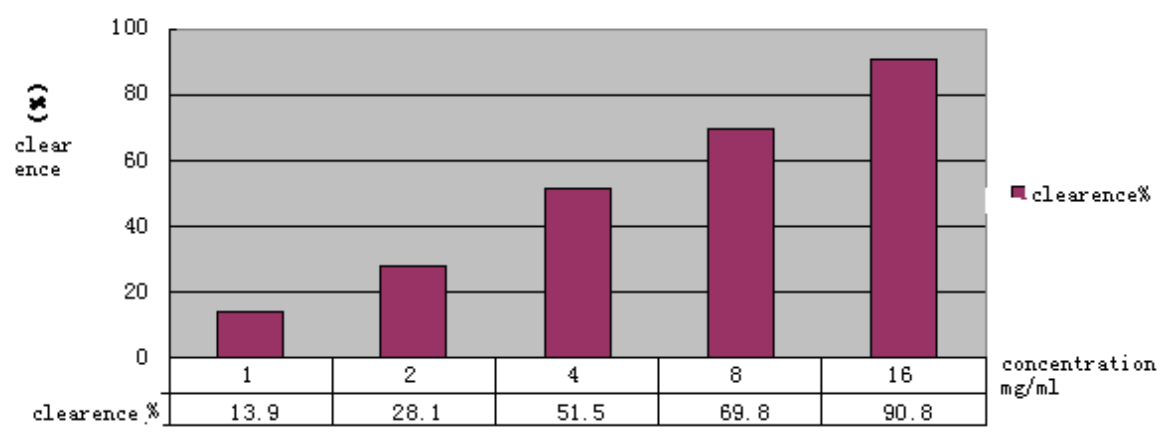

Figure1. The volatile oil of different concentrations of hydroxyl free radical clearance

\subsection{Bacteriostasis of Zhaoqing Yellow Fruit Oil}

The perfume solutions of different concentrations all showed inhibitory effects for the different types of bacteria. The bacteriostatic ring became small with essential oil concentrations decreasing. Both of them occurred a positive correlation. The inhibitory effect on Staphylococcus aureus and Bacillus subtilis were better, and the minimum inhibitory concentration (MIC) was $0.3125 \%$ for concentration of volatile oil. The antibacterial effect on Escherichia coli was relatively smaller, minimum inhibitory concentration (MIC) was $0.625 \%$ for concentration of volatile oil( Seeing Figure 2 and table 3).

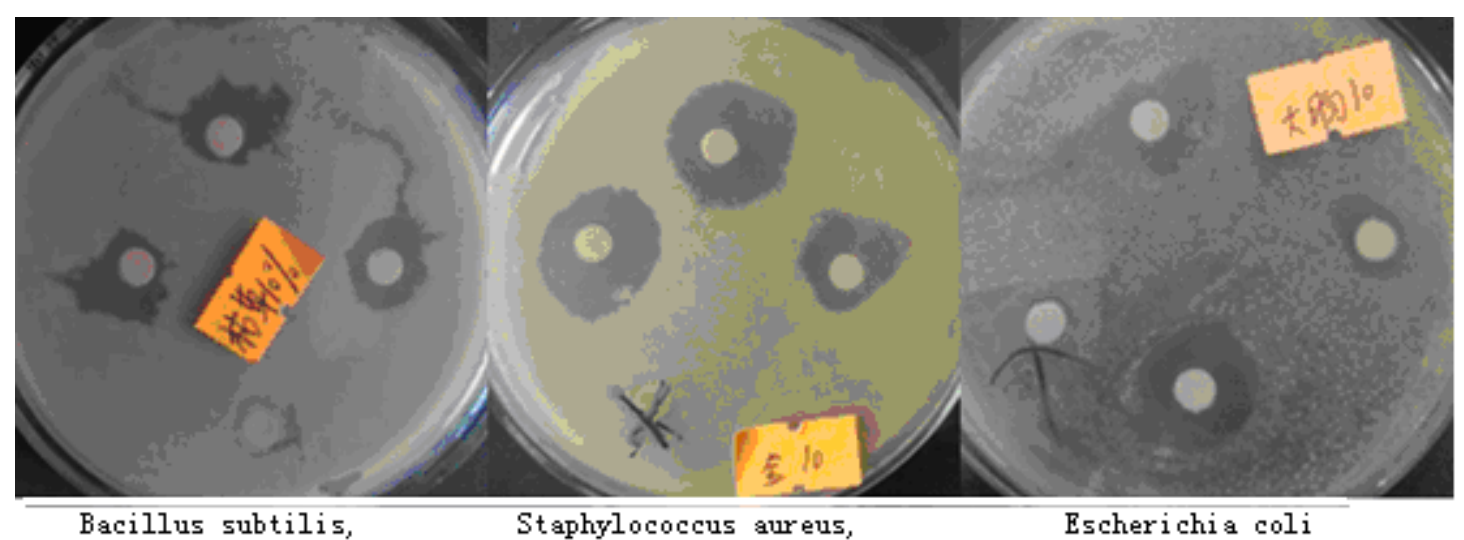

Fig2. Zhaoqing yellow fruit antibacterial effect of essential oil

Table3. The diameter of inhibition zone $(\mathrm{mm})$ of different concentrations of volatile oil to each test bacterium

\begin{tabular}{|l|l|l|l|l|l|l|l|l|}
\hline $\begin{array}{l}\text { Concentration } \\
\text { of volatile oil } \\
\text { percent }\end{array}$ & 10 & 5 & 2.5 & 1.25 & 0.625 & 0.312 & 0.156 & acetone \\
\hline $\begin{array}{l}\text { Bacillus } \\
\text { subtilis }\end{array}$ & $15.36 \pm 0.24$ & $12.70 \pm 0.17$ & $11.17 \pm 0.28$ & $11.00 \pm 0.47$ & $10.50 \pm 0.2$ & $9.58 \pm 0.29$ & ---- & -- \\
\hline $\begin{array}{l}\text { Escherichia } \\
\text { coli }\end{array}$ & $11.80 \pm 0.23$ & $11.00 \pm 0.58$ & $10.67 \pm 0.28$ & $10.42 \pm 0.32$ & $9.75 \pm 0.375$ & ---- & ---- & --- \\
\hline $\begin{array}{l}\text { Staphylococcus } \\
\text { aureus }\end{array}$ & $16.57 \pm 0.44$ & $15.17 \pm 0.42$ & $14.06 \pm 0.56$ & $11.75 \pm 0.28$ & $11.33 \pm 0.43$ & $11.17 \pm 0.14$ & ---- & -- \\
\hline
\end{tabular}

--- noninhibited

\subsection{Comparison of Inhibit Cancer Cells on the Perfume}

Recovery of mouse pancreatic cancer cells with each containing an equal amount of perfume and 95\% ethanol culture flask culture observed and recorded each time the two culture flask cancer cell number 


\section{Qiaohui Liang et al.}

and cancer cell death. It was found that the perfume on cancer cell growth inhibition was better than the control when comparing with $95 \%$ ethanol anti cancer effect $(\mathrm{P}<0.05$, Table 4$)$.

Table4. Comparison of inhibit cancer cells on the perfume and $95 \%$ ethanol $(X \pm S) P$

\begin{tabular}{|l|l|l|l|l|l|l|}
\hline \multicolumn{2}{|l|}{ Time of incubation } & $0 \mathrm{~h}$ & $1 \mathrm{~h}$ & $4 \mathrm{~h}$ & $18 \mathrm{~h}$ & $24 \mathrm{~h}$ \\
\hline \multirow{4}{*}{$95 \%$ ethanol } & cell $(\mathrm{n})$ & $95 \pm 12$ & $84 \pm 09$ & $73 \pm 07$ & $42 \pm 08$ & $21 \pm 03$ \\
\cline { 2 - 7 } & mortality $(\%)$ & --- & 11.58 & 13.10 & 42.46 & 50 \\
\hline \multirow{3}{*}{ perfume } & cell $(\mathrm{n})$ & $60 \pm 04$ & $50 \pm 05$ & $40 \pm 06$ & $20 \pm 02$ & 0 \\
\cline { 2 - 6 } & mortality (\%) & --- & 16.67 & 20 & 50 & 100 \\
\hline
\end{tabular}

\section{DisCUSSION}

The studies showed that origin of climate and environment is different, it could affect the internal composition difference (Liu Youjie. 2014). So it is necessary to study the characteristics of the essential oil from yellow skin peel in Guangdong zhaoqing region in order to explore the pharmacological effects for making full use of the yellow skin fruit and knowing it's function.

On the volatile oil yields, the extracting rates of zhaoqing wampee fruit were close to that of chinese wampee from guangzhou fruit for $0.4 \%$ ( Li Ruizhen et al, 2007), however, slightly higher than that of volatile oil production from Hainan fruit of Chinese wampee for $0.39 \%$ (Tang Wenning et al,2002 ).There is no much difference. The main difference lies in the composition and contents. Zhaoqing specific wampee fruit essential oil composition is mainly phellandrene (36.97\%), terpinen-4-ol (17.58\%), sabinene hydrate (13.76\%), and Guangzhou fruit of Chinese wampee volatile oil content higher is terpinen-4-ol (21.06\%), gamma Terpinene (12.9\%), 3, 7, 7 - trimethyl - bicyclo [4.1.0] has - 2 ene (29.3\%); Haikou wampee fruit essential oil content is higher for terpinen-4-ol (28.549_\%\%), sabinene (14.53 9\%), gamma Terpinene (4 4_.868_\%\%). Comparing that of Chinese wampee, although most of the components same, its essential oil contents is different. It is the first time to analyze Zhaoqing wampee of volatile oil for its pharmaceutical function and application. Preliminary studies on volatile oil of Zhaoqing specific wampee fruit antioxidant showed that volatile oil on hydroxyl free radical had a good scavenging effect in a certain concentration range; with increasing of concentration of volatile oil its scavenging of hydroxyl radical rate was raising. There was a good correlation in $1-16 \mathrm{mg} / \mathrm{ml}$ range for positive correlation. in the essential oil concentration and clear effects Hydroxyl radical scavenging rate is an important indicator of the antioxidant properties of substance. It is found that zhaoqing specific wampee fruit volatile oil had a certain resistance to oxidation through the point of view of the experimental results (Jiang Zitao et al., 2012, Chen Sijia et al., 2015, Zhang Fuping et al., 2013). And the result of hydroxyl radical scavenging rate increased as increasing the concentration of volatile oil in a certain range of concentration was also conformed to that of the references for $0.72 \mathrm{mg} / \mathrm{ml}$ in lemon essential oil(Liu Yinhua,2009). And it was proved that the salicylic acid method used to study the caraway seed oil on hydroxyl free radical scavenging rate influence could achieve better hydroxyl free radical scavenging effect (Chen Sijia, 2015). The volatile oil (essential oil) of specific yellow peel in Zhaoqing on hydroxyl free radical scavenging effect is relatively weak in the two kinds of essential oils. Currently, the yellow fruit essential oils in antioxidant research haven't been reported. This experimental result showed that the scavenging action to hydroxyl free radical as indicators of antioxidant properties would be an important antioxidant indicator. And it would provide a theoretical basis for the use of wampee fruit.

It is known that hydroxyl radical is the active oxygen of the human body, which have antioxidant capacity. The inhalation of essential oils existed in the different plant roots, stems, leaves, flowers, fruit had some different effects (Chen Sijia et al, 2015), and pure natural essential oil as a volatile oil contained large amounts of terpenes, aldehydes, lipids, ketones, phenols or phenolic ether terpene hydrocarbon mono olefin and diene, in which single graphene had antiseptic, analgesic and also could increase the body's immune function. It was reported that a half proportion of graphene could be anti-inflammatory, antipruritic, anti fungal and balance hormone secretion(Liang Qiaohui et al, 2015) Therefore, yellow volatile oil which used as a spice for the preparation of perfume is valuable in theoretical and practical significance. The successful wampee fruit essential oil perfume has a better smell of wampee fruit, and the long persistence in the filter paper holding incense is better than the commercially available Mayerton perfume. 
The knowledge of pharmacological activity on wampee volatile oil was not clear, and antimicrobial activity of the volatile oil on zhaoqing wampee fruit have not been reported up to now. The experiment showed that the inhibited bacteria effects of the yellow volatile oil increased with the contents increasing, it showed that the oil would become a potential antibacterial drug, but its effective antimicrobial components in isolation, identification and bacteriostasis mechanism need to be a further exploration.

The research has been supported by fund of Guangdong Provincial Department of science and technology [2011] 101 as well as the university student innovation project (write number?)

Thanks for support of the project from provincial scientific department [2011] no. 101 and college students' innovative project funding (201310580003).

\section{REFERENCES}

[1] Chen Sijia, Liu Yali, Zhang Chen, Zhang Xiangyu. Study on antioxidant activity of essential oils of lemon [J]. Journal of practical oral medicine, 2015,31, (2): 70 -66

[2] Jiang Zitao, sun Morey, Li Rong. Microwave assisted extraction and antioxidant activity of the essential oil from the aromatic oil. Chinese seasoning, [J] 2012, 37 (4): 112 - 19

[3] Li Ruizhen, Liao Huawei, Chen Feiyuan. Guangzhou yellow fruit essential oil composition, Journal of Guangdong Pharmaceutical University,[J]. 2007, 23 (2): 143 - 141

[4] Liang Qiaohui; Zheng Peijun; Huang Guibin; Wan Yi Huang; Anne down; Li Chongbi. Zhaoqing characteristics of wampee fruit essential oil components and scavenging hydroxyl free radical ability. Agriculture and technology,[J] 2015,35 9).25-27.

[5] Liu Yinhua. Research status of essential oil of Chinese herbal medicine Modern Chinese medicine, [J].2009,29 (1)68-70.

[6] Liu You. Investigation, collection and utilization of wampee germplasm resources in Fujian: Fujian Kasetsart University, [D] 2014

[7] Mei Jia Qi. Function of essential oil perfume research progress and application. Guangdong Xin De aromatic technology studio[a]. The tenth Chinese flavor Symposium Proceedings [C]. Hangzhou: China spice fragrance cosmetics industry association, 2014:03-308.

[8] Su Xiufang; Huang Lijuan; Feng Peizhen. Nutlets of Clausena anisum olens constituents and antimicrobial activity of essential oils from . food science,[J] 2011,32 3): 30-32.

[9] Tang Wenning, Kang literature, Mu Shuzhen, Hao Xiaojiang. Research and development of wampee fruit essential oil components of natural products, [J] 2002, 14 (2): 26 - 28

[10] The State Administration of traditional Chinese medicine "Chinese Materia Medica" Editing Committee of Chinese materia medica. (First Edition) [M]. Shanghai: Shanghai science and Technology Press, 999

[11] Zhang Fuping, Tang Yanji, Liu Xiaozhen, Chen Huanbin, Chen Xuefen. Yellow leaf flavonoids antioxidant activity of South China.Journal of Agricultural Sciences, [J]. 2013, 44 (8): 1343-146.

[12] Zhang Zhi; Teng Xiangjin; Hao Zaibin. Thin layer chromatography analysis of Stevia glycoside. China spices, [J] 2009; 34 (3: 94-98.

[13] Xiao-Hua Zhou, Nancy A. Obuchowski, Donna K. McClish, Statistical Methods in Diagnostic Medicine, Second Edition, Wiley Series in Probability and Statistics, 1 APR 2011.

[14] XingYan Bian, MTT colorimetric method and its application. foreign medical clinical biochemistry and Laboratory Medicine Sciences,[R] 1998, 1 (2): 83-85 